\title{
Linear and Nonlinear Noise Characterisation of Dual Stage Broadband Discrete Raman Amplifiers
}

\author{
M. A. Iqbal, M. A. Z. Al-Khateeb, L. Krzczanowicz, I. D. Philips, P. Harper and W. Forysiak
}

\begin{abstract}
We characterise the linear and nonlinear noise of dual stage broadband discrete Raman amplifiers (DRAs) based on conventional Raman gain fibres. Also, we propose an optimised dual stage DRA setup that lowers the impact of nonlinear noise (generated in the amplifier) on the performance of a transmission link (with $100 \mathrm{~km}$ amplifier spacing). We numerically analyse the design of a backward pumped cascaded dual stage 100nm DRA with high gain $(\sim 20 \mathrm{~dB})$ and high saturated output power $(>23 \mathrm{dBm})$. We show that the noise figure (NF) of the dual stage DRA is mainly dominated by the first stage irrespective of the type of gain fibre chosen in the second stage, and we also demonstrate that optimising the length and the type of Raman gain fibre can have significant impact on the size of inter/intra signal nonlinearities generated. Here, we report a theoretical model to calculate the nonlinear noise power generated in transmission spans with dual stage DRAs considering piecewise signal power evolution through the Raman gain fibres. The predicted signal to noise ratio (SNR) performances are calculated from the combined contributions from NF and nonlinear product power obtained using the proposed analytical model for transmission systems deployed with $100 \mathrm{~km}$ transmission span compensated by different dual stage DRAs. Finally, an optimised IDF $6 \mathrm{~km}$ - SMF $10 \mathrm{~km}$ dual stage configuration has been identified using the theoretical model, which allows maximum SNR of $14.6 \mathrm{~dB}$ at $1000 \mathrm{~km}$ for 1THz Nyquist WDM signal and maximum transmission reach of $3400 \mathrm{~km}$ at optimum launch power assuming $8.5 \mathrm{~dB}$ HD-FEC limit of the Nyquist PM-QPSK signal.
\end{abstract}

Index Terms - Optical fibre, nonlinear effects, optical fibre communication, optical amplifiers.

\section{INTRODUCTION}

$\mathrm{D}$ ISCRETE Raman amplifiers are known for their benefits over conventional erbium doped fibre amplifiers (EDFAs) in terms of arbitrary gain bandwidth, better design of gain shape and continuous gain spectrum [1-4]. Unlike distributed Raman amplifiers which use transmission fibre as

This work was supported by UK EPSRC grants ToM3 (EP/M009092/1) and PEACE (EP/L000091/1). (Corresponding author: Md Asif Iqbal)

The authors are with Aston Institute of Photonic Technologies, Aston University, Birmingham, B4 7ET, U.K. (e-mail: iqbalm7@aston.ac.uk; elkhateeb.eng@gmail.com;

1.krzczanowicz@aston.ac.uk; i.phillips@aston.ac.uk; p.harper@aston.ac.uk; w.forysiak@aston.ac.uk).

Copyright (c) 2015 IEEE. Personal use of this material is permitted. However, permission to use this material for any other purposes must be obtained from the IEEE by sending a request to pubs-permissions@ieee.org. the Raman gain medium, DRAs use separate gain fibre with smaller mode field diameter and higher Raman gain efficiency than conventional standard single mode fibre (SMF) to provide higher pump conversion efficiency. Dispersion compensating fibre (DCF) and highly nonlinear fibre (HNLF) are the most commonly used silica based Raman gain fibres for DRA applications. They are chosen for their high Raman gain efficiency due to high Germanium $(\mathrm{Ge})$ concentration in the silica core [5-8]. Inverse dispersion fibre (IDF) has recently been introduced as an alternative to conventional DCF for in-line dispersion compensation and can also be used in DRAs $[9,10]$. These conventional Raman gain fibres (DCF, HNLF, and IDF) have higher nonlinear properties than SMF due to higher nonlinear refractive index. The main challenges of using these as gain fibres, are double Rayleigh backscattering (DRS) induced multipath interference (MPI) $[11,12]$ and Kerr-induced nonlinear impairments $[13,14]$. In DRAs, DRS-induced MPI noise significantly deteriorates the optical signal to noise ratio (OSNR) and sets an upper limit on maximum achievable net gain to $15-17 \mathrm{~dB}$ from a single stage amplifier depending on the application [2]. The accumulation of DRS-induced noise can be suppressed by: (1) dividing the gain fibre into two stages with an optical isolator [15] and (2) using a gain flattening filter at appropriate position [16]. However, the main drawbacks of these schemes are: the high pump powers needed to amplify both stages reusing the residual pumps $[17,18]$ and high gain variations across the amplification bandwidth due to poor control of pump powers in each stage. Moreover, nonlinear phase shift (NPS) due to high path average power can degrade the transmission performances due to the use of long lengths of the same gain fibre [14].

Using a dual stage DRA with mid-stage isolator and independent pumping in each stage can increase the MPIlimited single stage gain and overall saturated output power of the amplifier. However, to minimise penalty due to linear and nonlinear noise, there is a trade-off between the type of Raman fibre used (which dictates the nonlinear refractive index), the fibre length, and which stage in the design we are considering.

In our previous study [19], we numerically analysed the design of a dual stage DRAs in terms of NF and self-phase modulation (SPM) induced nonlinear penalty, allowing the optimum fibre type to be selected. In this paper, we extend our previous results and model the linear and nonlinear noise 
characteristics of broadband $(100 \mathrm{~nm}), \sim 20 \mathrm{~dB}$ average net gain, $>23 \mathrm{dBm}$ saturated output power dual stage DRAs using combinations of conventional Raman gain fibres IDF, HNLF, DCF and finally we also consider the performance of SMF. We characterise the linear noise in terms of NF taking into consideration both amplified spontaneous emission (ASE) and DRS induced noise. Moreover, SPM-induced NPS and nonlinear Kerr product power are calculated to determine the nonlinear performances of different DRA configurations. Finally, a cascaded combination of IDF-SMF as first - second stage is identified to provide the minimum linear and nonlinear noise accumulation (in the 4 cases that we have studied) but at a cost of lower pump efficiency due to low Raman gain efficient SMF in the second stage. We also demonstrate that pump power efficiency can be significantly improved by introducing IDF in the second stage in an IDFIDF based DRA with $\sim 0.5 \mathrm{~dB}$ SNR degradation when compared to the optimal IDF-SMF configuration.

\section{Dual Stage DRA Design}

The configuration of dual stage DRA is shown in Fig. 1. The first stage consists of a Raman gain fibre backward pumped with five cascaded first-order depolarised Raman pumps $(1425,1444,1462,1476$ and 1508nm). The five pump wavelengths were enough to achieve gain flatness $<3 \mathrm{~dB}$ across $100 \mathrm{~nm}$.

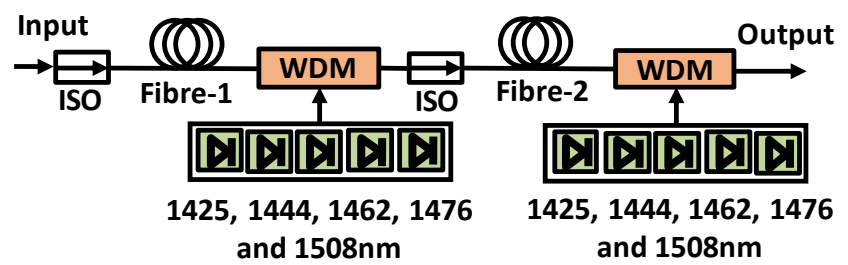

Fig. 1. Dual stage DRA consisting of two Raman gain fibres and a mid-stage isolator (ISO = isolator, WDM = wavelength division multiplexer)

At the input, broadband WDM signal consisting of CW signals at $5 \mathrm{~nm}$ spacing and $-20 \mathrm{dBm}$ per channel over $100 \mathrm{~nm}$ $(1525-1625 \mathrm{~nm})$ was considered for the calculation of gain and NF through numerical modelling of different DRA configurations. An input isolator was also considered to separate the amplification stage from the transmission fibre. The target average net gain from the first stage was set to $14 \mathrm{~dB}$ considering maximum pump power per wavelength of $500 \mathrm{~mW}$. Only IDF, HNLF and DCF were considered in the first stage due to a combination of higher Raman gain efficiency, and relatively low input signal power. The $14 \mathrm{~dB}$ net gain in the first stage ensures reasonably high gain and negligible Rayleigh backscattering induced OSNR penalty (less than $0.1 \mathrm{~dB}$ ) over the amplification bandwidth for all three gain fibre types at the optimised length. At the output of the first stage, a WDM coupler injects the pumps (in the backward direction) into the gain fibre and separates the amplified signals (propagating in the forward direction) into the output port. The lengths of gain fibres in the first stage were varied depending of fibre type as follows: IDF (6-14km),
HNLF (2-5km) and DCF $(5-13 \mathrm{~km})$. Similarly, the second stage was formed by another gain fibre pumped by the same set of pump wavelengths as in the first stage. In general, fine control of gain flatness and overall net gain could be achieved thanks to the independent pumping of each stages; however, we note overall gain flatness could be further improved by choosing different sets of (interleaved) pump wavelengths in the two stages. A mid-stage isolator with $1 \mathrm{~dB}$ insertion loss is used to isolate the two stages and also restrict the generation of DRS-induced MPI noise along the gain fibres. The second stage is considered as a booster amplifier with only $7 \mathrm{~dB}$ net gain (including $1 \mathrm{~dB}$ loss compensation of the mid-stage isolator) in order to obtain overall average signal net gain of $\sim 20 \mathrm{~dB}$ from the dual stage DRA. All three Raman gain fibres (IDF, HNLF and DCF) and SMF were considered as possible second stage gain fibres to characterise the accumulation of linear and nonlinear noise along the various dual stage DRA combinations.

\section{Numerical Characterisation OF Single Stage DRA}

The complete numerical model for the evolution of signals, pumps and different noise components is based on a standard average power model $[4,20,21]$. In this model, all the important features such as: fibre attenuation, pump depletion, ASE noise, DRS, Raman interactions between pump-to-signal, pump-to-pump and signal-to-signal were considered.

Wavelength dependent attenuation, DRS and Raman gain coefficients were considered in the model for all fibre types. Comparisons of Raman gain coefficient $\left(g_{R}\right)$ spectra with
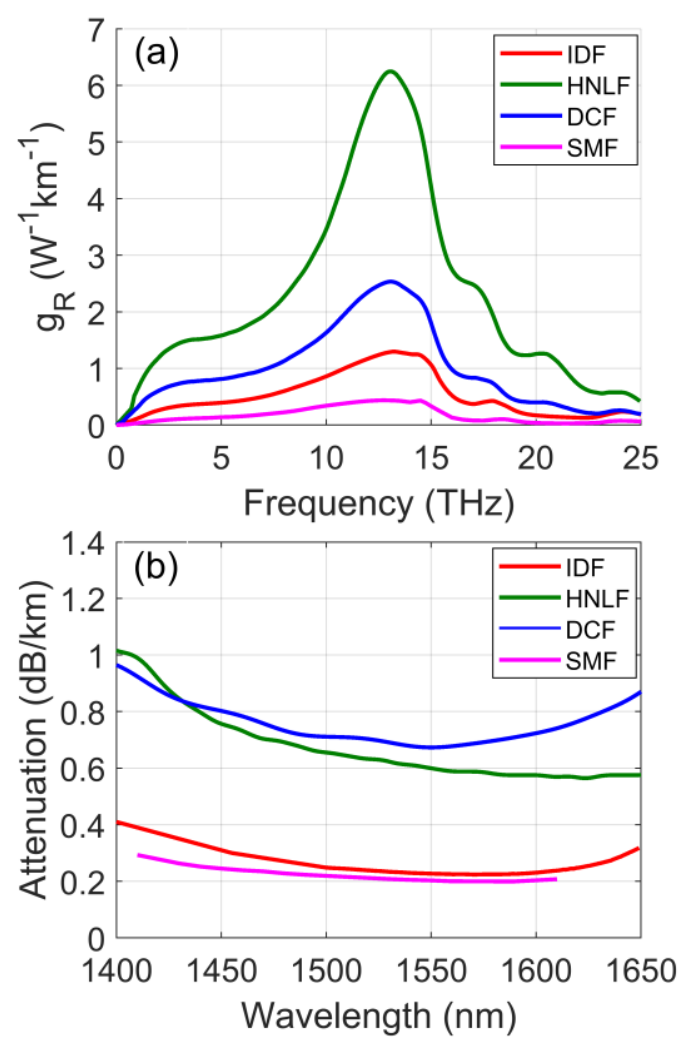

Fig. 2. Comparison of (a) Raman gain coefficients and (b) fibre attenuation coefficients among IDF, HNLF, DCF and conventional standard SMF 
respect to $1450 \mathrm{~nm}$ pump and attenuation coefficient profiles of different fibre types are shown in Fig. 2(a) and 2(b) respectively [22-24]. A standard SMF was chosen here to compare the performance with IDF, HNLF and DCF.

The peak values of $g_{R}$ for IDF, HNLF, DCF and SMF are $1.3,6.3,2.5$ and $0.43 \mathrm{~W}^{-1} \mathrm{~km}^{-1}$, respectively. SMF has the lowest loss over the entire wavelength region, whereas, IDF has the lowest attenuation both in signal and pump bands among the three Raman gain fibres and DCF shows the highest attenuation. A summary of the different parameters is given in Table I. HNLF has the lowest effective area of $10.2 \mu \mathrm{m}^{2}$ and largest nonlinear coefficient of $17.84 \mathrm{~W}^{-1} \mathrm{~km}^{-1}$ which is almost two and five times larger than DCF and IDF respectively. The Rayleigh scattering coefficient of SMF is the smallest among all. IDF also has much lower Rayleigh scattering coefficient $\left(1.2 \times 10^{-4} \mathrm{~km}^{-1}\right)$ than DCF and HNLF. All three Raman gain fibres have negative dispersion values in the signal band with DCF having the highest of -100 $\mathrm{ps} / \mathrm{nm} / \mathrm{km}$ in contrast to SMF dispersion of $16.5 \mathrm{ps} / \mathrm{nm} / \mathrm{km}$.

TABLE I

SUMMARY OF FIBRE PARAMETERS USED IN THE NUMERICAL CHARACTERISATION OF DIFFERENT DRAS

\begin{tabular}{|c|c|c|c|c|c|}
\hline Parameters & Unit & IDF & HNLF & DCF & SMF \\
\hline Attenuation $\left(\alpha_{1550}\right)$ & $\mathrm{dB} / \mathrm{km}$ & 0.23 & 0.6 & 0.67 & 0.2 \\
\hline Attenuation $\left(\alpha_{1450}\right)$ & $\mathrm{dB} / \mathrm{km}$ & 0.31 & 0.76 & 0.8 & 0.25 \\
\hline $\begin{array}{c}\text { Effective area } \\
\left(\mathrm{A}_{\text {eff }}\right) @ 1550 \mathrm{~nm}\end{array}$ & $\mu \mathrm{m}^{2}$ & 31 & 10.2 & 19 & 80 \\
\hline $\begin{array}{c}\text { Peak Raman gain } \\
\text { coefficient }\left(\mathrm{g}_{\mathrm{R}}\right)\end{array}$ & $\mathrm{W}^{-1} \mathrm{~km}^{-1}$ & 1.3 & 6.3 & 2.5 & 0.43 \\
\hline $\begin{array}{c}\text { Rayleigh scattering } \\
\text { coefficient }\left(\kappa_{1550}\right)\end{array}$ & $\left(\mathrm{km}^{-1}\right) \times 10^{-4}$ & 1.2 & 9.2 & 7.1 & 0.43 \\
\hline $\begin{array}{c}\text { Nonlinear } \\
\text { coefficient }\left(\gamma_{1550}\right)\end{array}$ & $\mathrm{W}^{-1} \mathrm{~km}^{-1}$ & 3.53 & 17.84 & 8.43 & 1.4 \\
\hline Dispersion $\left(\mathrm{D}_{1550}\right)$ & $\mathrm{ps} / \mathrm{nm} / \mathrm{km}$ & -44 & -20.7 & -100 & 16.5 \\
\hline
\end{tabular}

The accumulation of linear and nonlinear noise were characterised first in the single stage configuration to determine the optimum gain fibre length within the set maximum pump power $(500 \mathrm{~mW} /$ wavelength) and target net gain of $14 \mathrm{~dB}$. The accumulation of linear noise was characterised in terms of NF over $100 \mathrm{~nm}$ amplification bandwidth at $-20 \mathrm{dBm}$ input signal power per channel. Both ASE noise and DRS contributions were included for NF calculation $[25,26]$. The nonlinear noise was calculated in terms of NPS by evaluating the signal power profile along the gain fibre length for each signal wavelength according to (1),

$$
\operatorname{NPS}(\lambda)=\gamma(\lambda) \int_{0}^{L} P(\lambda, z) d z
$$

where, $\gamma$ and $L$ represent the wavelength dependent nonlinear coefficient and length of particular gain fibre respectively and $P(\lambda, z)$ denotes the wavelength dependent power profile along the length $(z)$ of the amplifier [1].

Fig. 3 shows the characterised results for different lengths of IDF from $6 \mathrm{~km}$ to $14 \mathrm{~km}$. The overall net gain characteristics for different IDF lengths is shown in Fig. 3(a), in which gain variations $<2 \mathrm{~dB}$ are maintained by optimising the pump powers for different lengths using the Raman optimiser tool of VPItransmissionMaker ${ }^{\mathrm{TM}} 9.8$ which uses a genetic algorithm (GA) for pump power optimisation [27-29]. The minimum length of IDF that could provide $14 \mathrm{~dB}$ net gain with the maximum available pump power was $6 \mathrm{~km}$. However, increasing the Raman gain fibre length enhances the Raman interaction length between pump and signals and similar $14 \mathrm{~dB}$ net gain can be achieved with lower pump powers as shown in Table II. Doubling the amplifier span length from 6 to $12 \mathrm{~km}$ reduces the total pump power by $370 \mathrm{~mW}(\sim 34 \%)$. The maximum fibre length was limited to $14 \mathrm{~km}$ because beyond that point fibre attenuation begins to dominate over the net gain and the required pump power increases.

TABLE II

PUMP POWERS USED WITH DIFFERENT LENGTHS OF IDF

\begin{tabular}{|c|c|c|c|c|c|}
\hline \multirow{2}{*}{ Pumps } & \multicolumn{5}{|c|}{ Pump powers (mW) } \\
\cline { 2 - 6 } & $6 \mathrm{~km}$ & $8 \mathrm{~km}$ & $10 \mathrm{~km}$ & $12 \mathrm{~km}$ & $14 \mathrm{~km}$ \\
\hline $1425 \mathrm{~nm}$ & 450 & 430 & 385 & 355 & 340 \\
\hline $1444 \mathrm{~nm}$ & 315 & 230 & 205 & 186 & 176 \\
\hline $1462 \mathrm{~nm}$ & 130 & 103 & 89 & 81 & 80 \\
\hline $1476 \mathrm{~nm}$ & 104 & 78 & 66 & 60 & 48 \\
\hline $1508 \mathrm{~nm}$ & 108 & 80 & 65 & 55 & 46 \\
\hline Total & 1107 & 921 & 810 & 737 & 690 \\
\hline
\end{tabular}

The calculated NFs for different fibre lengths are shown in Fig. 3(b). NF tilts from shorter to longer wavelength signals were observed for all the backward pumped DRA schemes due to pump-to-pump, signal-to-signal energy transfer and fibre loss profile. The NF tilt can be minimised by using bidirectional pumping [21,30], which is out of the scope of

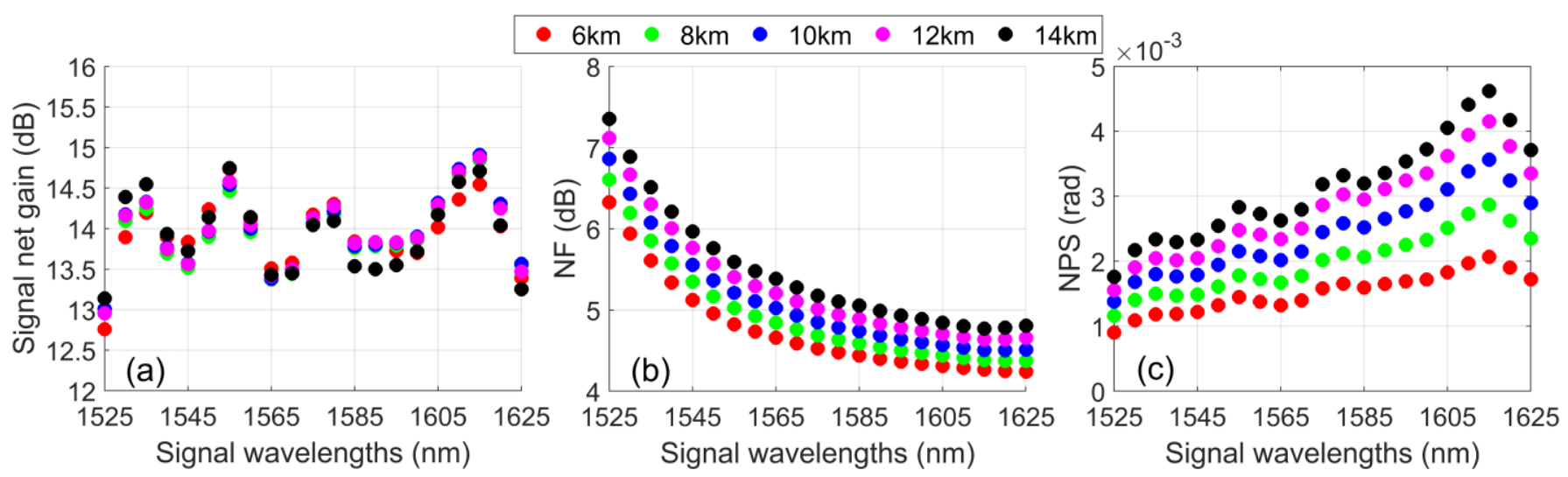

Fig. 3. Characterisation of IDF single stage based DRA with different lengths: (a) signal net gain (b) NF and (c) NPS 


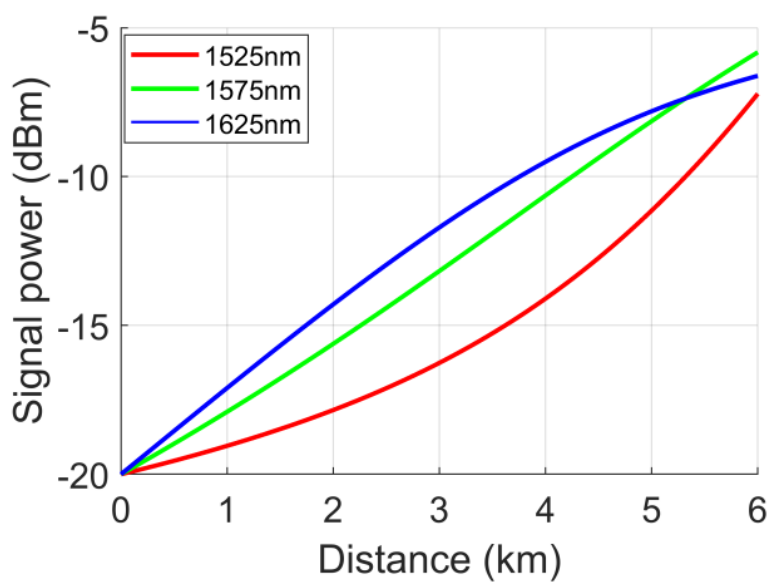

Fig. 4. Distribution of signal power at different wavelengths in IDF $6 \mathrm{~km}$ based single stage DRA

this paper. Using shorter lengths of gain fibre reduces ASE and DRS-induced noise and gives lower NF as can be seen from IDF 6km in Fig. 3(b). In Fig. 3(c), NPS is shown for all the signal wavelengths in the amplification band for different gain fibre lengths. Similar to NF, NPS is also higher when using longer IDF length. However, in contrast to NF, longer wavelength signals show higher NPS levels than shorter wavelengths due to greater average signal power along the span length in broadband DRAs, as can also be seen from the signal power evolution at different wavelengths in Fig. 4.

In Fig. 4, longer wavelength signals (1575 and 1625nm) propagate along the gain fibre with much higher power than that of lowest wavelength signal $1525 \mathrm{~nm}$, thanks to the pumpto-pump and signal-to-signal energy transfer from lower to higher wavelength components, this results in higher NPS at longer wavelength bands when compared to the NPS generated in the shorter wavelengths. The output power differences among the three wavelengths are due to net gain differences across the gain bandwidth as shown in Fig. 3(a).

Noise characterisation of the single stage DRA were also carried out for different lengths of HNLF $(2-5 \mathrm{~km})$ and DCF $(5-13 \mathrm{~km})$ considering the same $14 \mathrm{~dB}$ target net gain and maximum $3 \mathrm{~dB}$ gain ripple constraints. Similar trends in $\mathrm{NF}$ and NPS were observed in DCF and HNLF with the shortest
TABLE III

PUMP POWERS USED IN DIFFERENT SINGLE STAGE DRAS

\begin{tabular}{|c|c|c|c|}
\hline \multirow{2}{*}{ Pumps } & \multicolumn{3}{|c|}{ Pump power (mW) } \\
\cline { 2 - 4 } & IDF 6km & HNLF $2 \mathrm{~km}$ & DCF 5km \\
\hline $1425 \mathrm{~nm}$ & 450 & 300 & 385 \\
\hline $1444 \mathrm{~nm}$ & 315 & 190 & 345 \\
\hline $1462 \mathrm{~nm}$ & 130 & 90 & 105 \\
\hline $1476 \mathrm{~nm}$ & 104 & 63 & 92 \\
\hline $1508 \mathrm{~nm}$ & 108 & 68 & 94 \\
\hline Total & 1107 & 711 & 1021 \\
\hline
\end{tabular}

lengths of DCF $5 \mathrm{~km}$ and HNLF $2 \mathrm{~km}$ being the rational choice to achieve low NF and NPS in the respective cases. Gain and noise performance comparisons among these three different gain fibres (IDF, HNLF and DCF) at their respective best performing lengths are given in Fig. 5.

The overall average net gain of $\sim 14 \mathrm{~dB}$ over $100 \mathrm{~nm}$ bandwidth was maintained for each type of gain fibre as shown in Fig. 5(a). In Fig. 5(b), the NF of IDF $6 \mathrm{~km}$ and HNLF $2 \mathrm{~km}$ shows almost identical trend over the full amplification bandwidth whereas, DCF $5 \mathrm{~km}$ gives the worst NF with $>1 \mathrm{~dB}$ higher NF around shorter signal wavelengths, which is due to higher Rayleigh backscattering coefficient giving higher DRS induced MPI noise. Although HNLF $2 \mathrm{~km}$ has similar linear noise accumulation as IDF $6 \mathrm{~km}$, it shows worse NPS which is similar to DCF $5 \mathrm{~km}$ as shown in Fig. 5(c). Here, DCF has lower nonlinear refractive index than HNLF (Table I), however signal power over the longer length of DCF makes the NPS induced nonlinear penalty similar to HNLF $2 \mathrm{~km}$. On the other hand, IDF has the lowest nonlinear coefficient among all and shows the lowest NPS in Fig. 5(c). The pump powers used in each gain fibre type are shown in Table III.

From the above characterisation it can be seen that IDF $6 \mathrm{~km}$ gives the lowest nonlinear footprint on the propagating signals with lowest NPS values across the amplifier bandwidth and also shows similar lowest NF values as HNLF $2 \mathrm{~km}$.

\section{Characterisation of Dual Stage DRAs}

The noise accumulation in cascaded amplifiers is mainly dominated by the first stage amplifier [31, 32]. Usually higher net gain and lower noise first stage is chosen for multi-stage cascaded amplifier chain for overall lower noise accumulation.

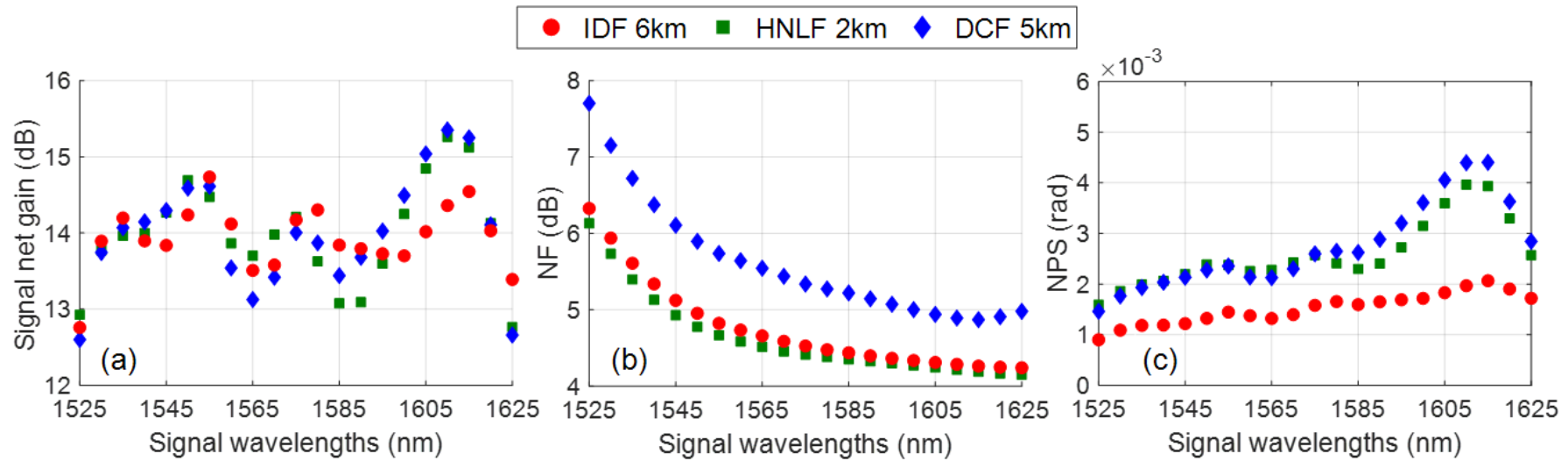

Fig. 5. Performance comparison of different single stage DRAs at optimum gain fibre lengths 


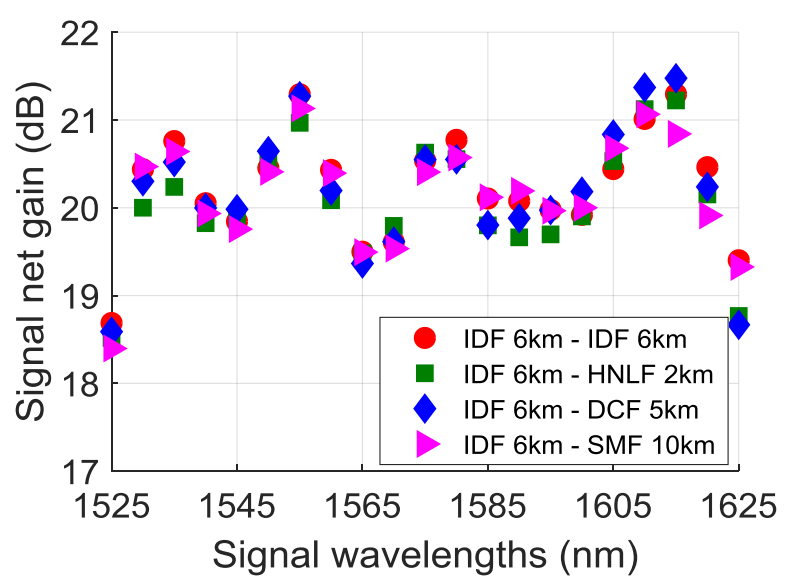

Fig. 6. Net gain comparison between different dual stage DRA configurations Consequently, since it was shown in Section III that IDF performs better than HNLF and DCF in the single stage DRA characterisation in terms of combined linear and nonlinear noise accumulation, here we focus only on IDF $6 \mathrm{~km}$ as the first stage in all the dual stage DRA configurations considered. For the second stage, all four types of gain fibres IDF, HNLF, DCF and SMF have been evaluated in terms of linear and nonlinear noise. The dual stage DRA configuration is shown in Fig. 1 in which an overall net gain of $20 \mathrm{~dB}$ is targeted for all the gain fibre combinations. In the second stage, the length of IDF, DCF and HNLF were chosen to be equal to their respective optimised single stage length, whereas the length of SMF $10 \mathrm{~km}$ was chosen in the second stage of IDF-SMF based DRA in order to enable a good balance between required pump powers and DRS-induced MPI noise.

Firstly, the net gain was kept fixed at $\sim 20 \mathrm{~dB}$ with $<3 \mathrm{~dB}$ gain ripple over $100 \mathrm{~nm}$ amplification bandwidth for all four (IDF $6 \mathrm{~km}$ - IDF $6 \mathrm{~km}$, IDF $6 \mathrm{~km}$ - HNLF 2km, IDF $6 \mathrm{~km}-$ DCF $5 \mathrm{~km}$ and IDF $6 \mathrm{~km}-\mathrm{SMF} 10 \mathrm{~km}$ ) dual stage DRA schemes with input power per channel of $-20 \mathrm{dBm}$ at the input of the first stage as shown in Fig. 6. Pump powers in the second stage were optimised using the same GA technique used in the single stage case and are given in Table IV. As expected, the HNLF $2 \mathrm{~km}$ based second stage DRA required least total pump power among all the schemes, whereas the SMF $10 \mathrm{~km}$ second stage based DRA required more than three times total pump power than HNLF $2 \mathrm{~km}$, because it has the lowest Raman gain coefficient and largest effective area among all the fibres considered here. The output power and net gain at different input signal power were also calculated for different DRAs as shown in Fig. 7, keeping the pump

TABLE IV

PUMP POWERS USED IN DIFFERENT DUAL STAGE DRAS

\begin{tabular}{|c|c|c|c|c|}
\hline \multirow{2}{*}{ Pumps } & \multicolumn{4}{|c|}{ Pump power (mW) } \\
\cline { 2 - 5 } & $\begin{array}{c}\text { IDF 6km } \\
2^{\text {nd }} \text { stage }\end{array}$ & $\begin{array}{c}\text { HNLF } 2 \mathrm{~km} \\
2^{\text {nd }} \text { stage }\end{array}$ & $\begin{array}{c}\text { DCF } 5 \mathrm{~km} \\
2^{\text {nd }} \text { stage }\end{array}$ & $\begin{array}{c}\text { SMF 10km } \\
2^{\text {nd }} \text { stage }\end{array}$ \\
\hline $1425 \mathrm{~nm}$ & 250 & 156 & 260 & 470 \\
\hline $1444 \mathrm{~nm}$ & 135 & 81 & 133 & 280 \\
\hline $1462 \mathrm{~nm}$ & 71 & 42 & 70 & 184 \\
\hline $1476 \mathrm{~nm}$ & 76 & 56 & 65 & 100 \\
\hline $1508 \mathrm{~nm}$ & 119 & 70 & 98 & 250 \\
\hline Total & 651 & 405 & 626 & 1290 \\
\hline
\end{tabular}

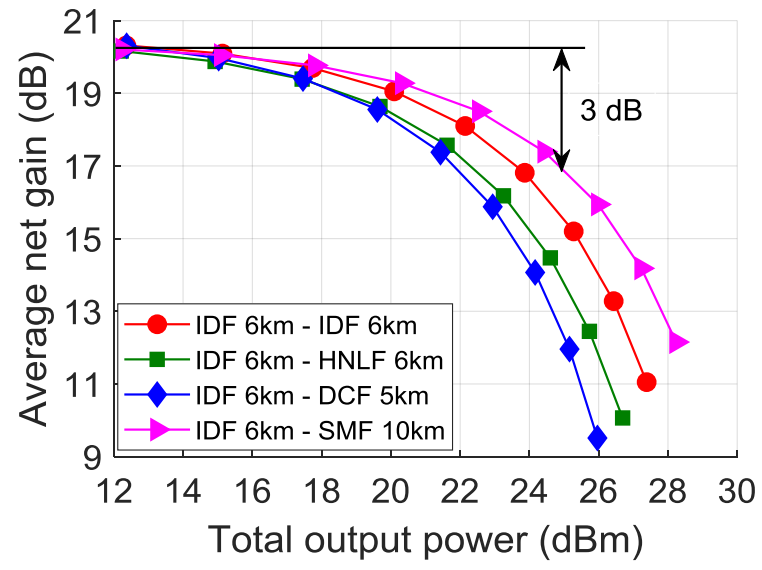

Fig. 7. Average net gain vs. total output power for different dual stage DRAs power fixed (Table IV).

Fig. 7 shows the characteristics of average net gain over $100 \mathrm{~nm}$ amplification bandwidth and total output power at different input signal power levels for different gain fibres in the second stage DRAs. The IDF $6 \mathrm{~km}-\mathrm{SMF} 10 \mathrm{~km}$ based DRA shows the maximum saturated output power of $\sim 24.5 \mathrm{dBm}$ calculated at the $3 \mathrm{~dB}$ lower average net gain point from the maximum $\sim 20 \mathrm{~dB}$. The saturated output power for SMF $10 \mathrm{~km}$ is $\sim 2.5 \mathrm{~dB}$ better than the worst performing DCF $5 \mathrm{~km}$ based second stage DRA. HNLF and DCF shows greater saturation due to stronger pump-to-pump and signal-to-signal Raman interactions.

The NF of dual stage DRAs with $20 \mathrm{~dB}$ average net gain are mainly dominated by the NF of the first stage (IDF 6km) with maximum $6.3 \mathrm{~dB} \mathrm{NF}$ at $1525 \mathrm{~nm}$ as shown in Fig. 8(a). All four
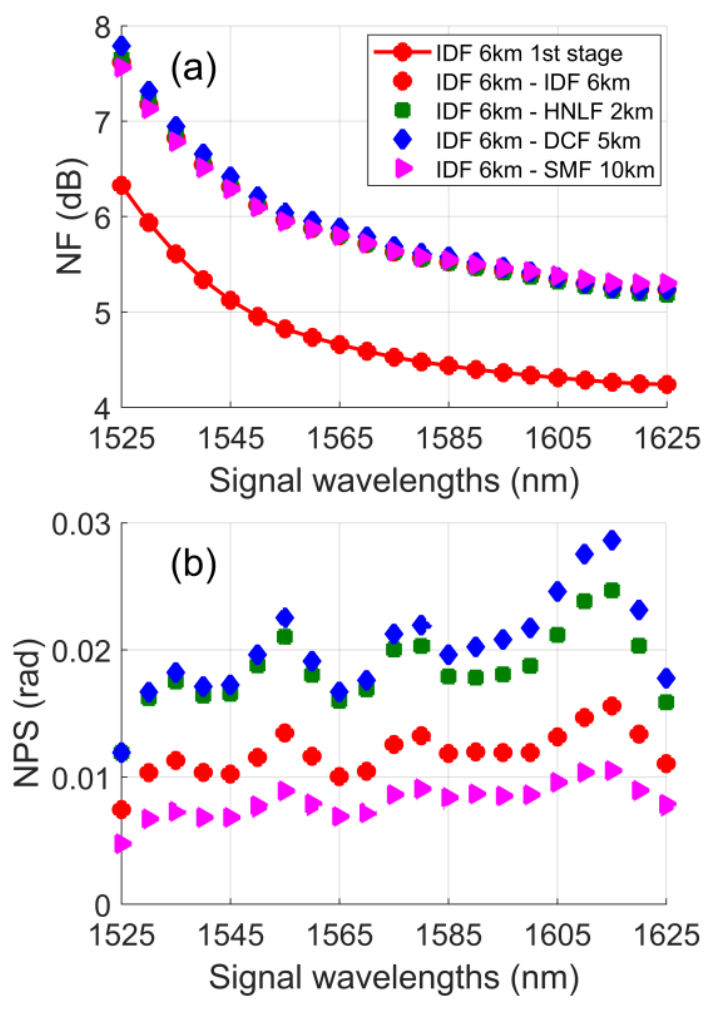

Fig. 8. Comparison of (a) NF and (b) NPS among different dual stage DRAs 
dual stage DRA schemes show quite similar results with about $1.3 \mathrm{~dB}$ maximum NF degradation versus the IDF $6 \mathrm{~km}$ based single stage DRA, so it is clear that the linear noise remains similar for all the cases irrespective of the second stage gain fibre type. In contrast, the nonlinear performances, i.e. NPS, varies strongly for the different gain fibre types as shown in Fig. 8(b). As expected, SMF shows the lowest nonlinearity with an average NPS of $0.008 \mathrm{rad}$. IDF provides only up to 1.5 times higher average NPS than SMF. Although HNLF has the shortest length $(2 \mathrm{~km})$ in the second stage, however it performs similar to DCF with an average NPS of 0.019 rad over the amplification bandwidth because of its highest nonlinear coefficient. The differences in NPS can also be explained by considering the signal power distribution along the dual stage DRA span lengths as shown in Fig. 9.

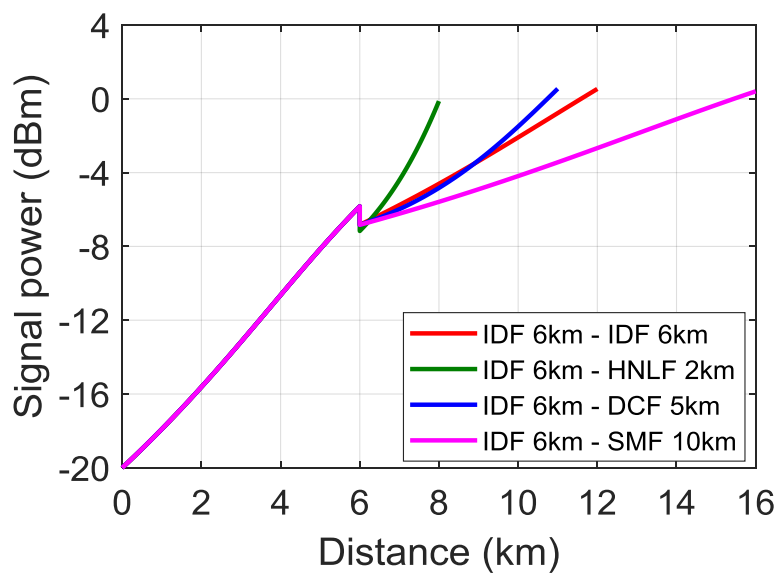

Fig. 9. Distribution of signal power at $1575 \mathrm{~nm}$ in different dual stage DRAs

In Fig. 9, the signal power distribution of the $1575 \mathrm{~nm}$ signal is shown for different dual stage DRAs. All the schemes have the same signal power profile in the common first stage and different power distributions in the second stage with different gain fibre types and lengths. The signal power along the length of the gain fibre and nonlinear coefficient both contribute to NPS as given in (1). Although the integrated signal power along DCF $5 \mathrm{~km}$ is lower than SMF $10 \mathrm{~km}$ in the second stage, the six times higher $\gamma$ parameter (Table I) of DCF provides overall 2.5 times higher average NPS than that of SMF. Similarly, although IDF $6 \mathrm{~km}$ and DCF $5 \mathrm{~km}$ have comparable power profiles, the NPS in DCF is greater because of its higher $\gamma$ parameter.

Therefore, choosing gain fibre with higher effective area as large as SMF can significantly reduce the NPS induced nonlinear penalty at the cost of pump power efficiency. IDF $6 \mathrm{~km}$ - IDF $6 \mathrm{~km}$ based dual stage DRA can significantly ( 2 times) improve the pump power efficiency (Table IV) with a small degradation in nonlinear penalty compared with the SMF $10 \mathrm{~km}$ based DRA.

The nonlinear interference among signals propagating along the DRA stages can be characterised more accurately than via NPS by analysing the nonlinear Kerr effects (SPM, cross phase modulation (XPM), or four wave mixing (FWM)). Analysing the system's nonlinearities (using XPM and FWM) highlights the impact of optical fibre nonlinear factor $(\gamma)$ as well as the effect of phase mismatching among signals due to the accumulation of chromatic dispersion $\left(\beta_{2}\right)$ along the system. When deploying DRAs in optical transmission links, the nonlinearities generated within the DRA could be higher in principle than the nonlinearities generated in the transmission fibre, which could therefore degrade the performance of the DRA-assisted link when compared to the conventional EDFAassisted link. Consequently, the inter-intra/signal nonlinear interference generated within the DRA should be minimised to maintain lower impact on the system performance.

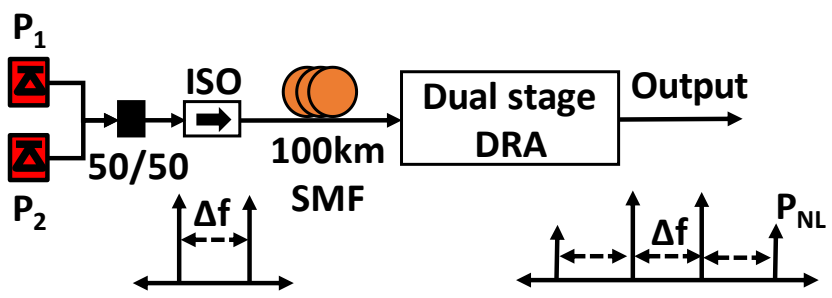

Fig. 10. Setup for nonlinear product power measurement in dual stage DRAassisted links (ISO $=$ isolator)

The nonlinear Kerr product power can be calculated by considering the propagation of two tunable continuous wave (CW) lasers into the system and obtaining the power of the nonlinear Kerr product at the output as a function of the CW laser frequency separation. Fig. 10 shows an example technique of measuring nonlinear Kerr product power generated from two $\mathrm{CW}$ lasers transmitted through a single transmission span of SMF $100 \mathrm{~km}$ and then amplified using a dual stage DRA to compensate for the SMF insertion loss $(20 \mathrm{~dB})$. The power of the two tunable $\mathrm{CW}$ lasers $\left(P_{1}\right.$ and $\left.P_{2}\right)$ were considered to be $3 \mathrm{dBm}$ (each) at the input of the transmission span. The power of the generated nonlinear product $\left(P_{N L}\right)$ was calculated at the output of the DRA (considering that the $\mathrm{CW}$ lasers were propagating at $1550 \mathrm{~nm}$ ) varying the frequency separation $(\Delta f)$ between the lasers from 1 to $20 \mathrm{GHz}$.

To calculate the nonlinear Kerr effect generated in DRAassisted transmission systems, we have derived an analytical approximation that predicts the nonlinear product power as a function of system parameters as given in (2). This expression is obtained from an analytical model that describes nonlinear Kerr effects in discretely amplified (i.e. EDFAs) [33-36] and distributed Raman amplification based transmission systems [37].

Equation (2) predicts the nonlinear Kerr product power $\left(P_{N L}\right)$ generated from the nonlinear interaction among up to three optical spectral waves with powers $P_{q}, P_{r}$ and $P_{s}$ that are

$$
P_{N L}=\left.\left(\frac{D}{3}\right)^{2} P_{q} P_{r} P_{s}\left[\frac{\sin ^{2}(N \overline{\Delta \beta L} / 2)}{\sin ^{2}(\overline{\Delta \beta L} / 2)} \mid \gamma_{\text {smf }} \frac{\mathrm{e}^{\left[\left(-\alpha+i \Delta \beta_{\text {span }}\right) L_{\text {span }}\right]}-1}{-\alpha+i \Delta \beta_{\text {span }}}+\gamma_{1} c_{1} \mathrm{e}^{\left[\left(-\alpha+i \Delta \beta_{\text {span }}\right) \frac{L_{\text {span }}}{2}\right]} \frac{\mathrm{e}^{\left[\left(g_{1}+i \Delta \beta_{1}\right) L_{1}\right]}-1}{g_{1}+i \Delta \beta_{1}}+\gamma_{2} c_{2} \mathrm{e}^{\left[\left(-\alpha+i \Delta \beta_{\text {span }}\right) \frac{L_{\text {span }}}{2}+\left(g_{1}+i \Delta \beta_{1}\right) L_{1}\right.}\right] \frac{\mathrm{e}^{\left[\left(g_{2}+i \Delta \beta_{2}\right) L_{2}\right]}-1}{g_{2}+i \Delta \beta_{2}}\right|^{2}
$$


propagating along a periodic dual stage DRA-assisted system. The nonlinear Kerr product power can be theoretically approximated in a closed form equation (shown in (2)) using a piecewise signal power profile approximation comprising a loss section from transmission fibre (normally SMF) and two gain sections of the dual-stage DRA; the contribution of each stage can be noticed, respectively, inside $|.|^{2}$ of (2). It is an extension of a theoretical model introduced previously that was used to approximate the nonlinear product power along a distributed Raman amplified link [37].

The spectral location of the generated nonlinear Kerr product is dependent on the relative spectral location of the mixing components as: $f_{N L}=f_{q}+f_{r}-f_{s}\left(f_{q}, f_{r}\right.$ and $f_{s}$ are the three interacting frequency components). In (2), $D$ represents the degeneracy factor that changes based on the number of the mixing components involved in the nonlinear process, and takes values of 1,3 , or 6 to represent the case of SPM $\left(f_{q}=f_{r}=f_{s}\right)$, XPM $\left(f_{q}=f_{r} \neq f_{s}\right)$, or FWM $\left(f_{q} \neq f_{r} \neq f_{s}\right)$, respectively. $L_{\text {span }}, L_{1}$, and $L_{2}$ in (2) represent the lengths of the transmission fibre span, the first and the second stage of the DRA, respectively. $\alpha, g_{1}$, and $g_{2}$ are the signal attenuation coefficient of the transmission fibre, the signal gain coefficient along the first stage and the second stage of the DRA (all in Neper/m), respectively. $N$ represents the number of transmission fibre spans and $\Delta \beta=\beta_{q}+\beta_{r}-\beta_{s}-\beta_{F}$ is the phase matching coefficient of each fibre section $\left(\Delta \beta_{\text {span, }} \Delta \beta_{1}\right.$, and $\left.\Delta \beta_{2}\right)$, which relates to the chromatic dispersion coefficient $\beta_{2}$ and the frequency separation between the mixing components: $\Delta \beta=-4 \pi^{2} \beta_{2}\left(f_{q}-f_{s}\right)\left(f_{r}-f_{s}\right) \cdot \overline{\Delta \beta L}=\Delta \beta_{\text {span }} L_{\text {span }}+\Delta \beta_{1} L_{1}+\Delta \beta_{2} L_{2}$ is the cumulative effective phase matching coefficient for the transmission fibre span, phase mismatching of the first stage DRA, and phase mismatching of the second stage DRA. In (2), $c_{1}$ represents the insertion loss of the isolator at the input of the first stage of the DRA (see Fig. (1)), while $c_{2}$ is the insertion loss of the mid-stage isolator.

From (2), it can be seen that the nonlinear product power $\left(P_{N L}\right)$ scales with the product of the powers of the mixing components $\left(P_{q} P_{r} P_{s}\right)$. The terms in the first squared brackets represent the oscillation in the nonlinear product power due to the periodicity of the signal power profile and phase matching along a periodic multi-span transmission system. The terms in the squared modulus $|.|^{2}$ represent the nonlinear product fields generated within the three fibres comprising the transmission span (first term), and the first (second term) and second stage (third term) of the DRA, respectively. It can be seen the nonlinear field generated per section is linearly dependent of the nonlinear factor of each fibre.

Fig. 11 shows the numerically simulated and theoretically calculated nonlinear product power using (2) as a function of frequency separation between the two $\mathrm{CW}$ lasers centred at $1550 \mathrm{~nm}$ as they propagate through the system described in Fig. 10. Here, we numerically simulate the propagation of two CW lasers by solving NLSE using split step FFT to measure the power of nonlinear product generated at the end of a transmission span compensated by different dual stage DRA configurations. The dual stage DRAs used in these

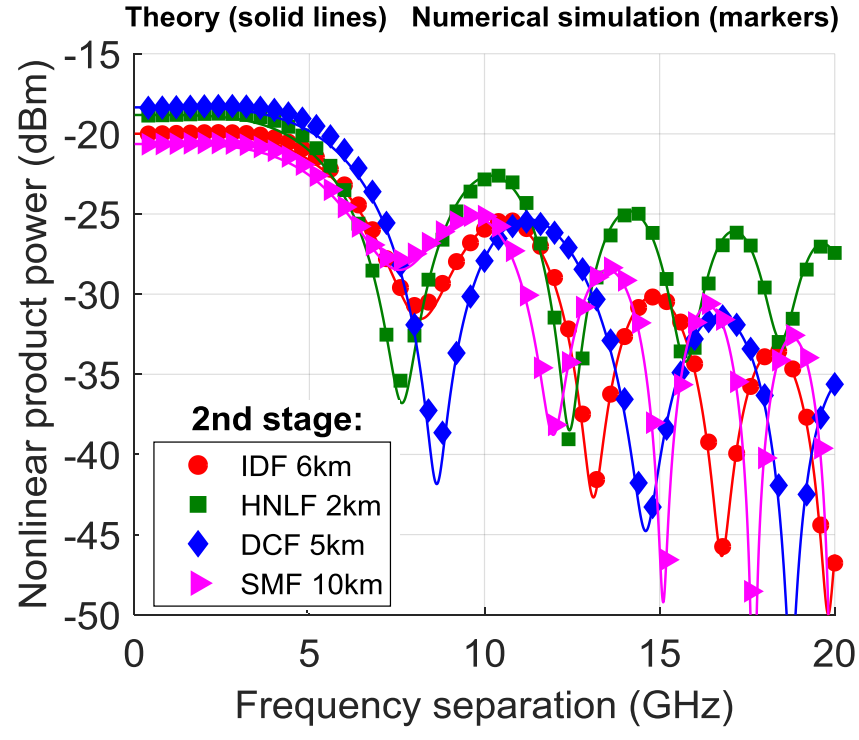

Fig. 11. Comparison among the theoretical and numerically simulated nonlinear Kerr product powers in different dual stage DRAs with fixed IDF $6 \mathrm{~km}$ based first stage and different second stage gain fibres

calculations correspond to the four cases shown in Fig. $9(6 \mathrm{~km}$ of IDF as first stage, and various types of fibres as second stages). The theoretical prediction in (2) shows a very good agreement with the numerically simulated results for Kerr induced nonlinear product powers validating the performance of our model considering large dispersion and nonlinear coefficient values of different Raman gain fibres. At low frequency separation $(<3 \mathrm{GHz})$, the nonlinear product power generated at the system output (shown in Fig. 10) achieves its highest value $(-18.4 \mathrm{dBm})$ when DCF $(5 \mathrm{~km})$ is used for a second stage gain fibre in the dual stage DRA, which is about $0.4 \mathrm{~dB}, 1.6 \mathrm{~dB}$ and $2.2 \mathrm{~dB}$ higher than when using HNLF $(2 \mathrm{~km})$, IDF $(6 \mathrm{~km})$ and SMF $(10 \mathrm{~km})$, respectively. At the lowest frequency separation, the nonlinear Kerr product powers from HNLF and DCF based DRAs show almost similar results, which is consistent with the similar NPS induced penalty at $1550 \mathrm{~nm}$ as shown in Fig. 8(b). As expected, a DRA based on SMF $(10 \mathrm{~km})$ in the second stage generates the lowest nonlinear product power, when compared to the other types of fibre; this is due to its lower nonlinear coefficient. At higher frequency separation ( $>3 \mathrm{GHz}$ ), the Kerr product power degrades and oscillates as a function of the frequency separation (when $\Delta \beta_{x x}>>\alpha$ ). This degradation and oscillation in Kerr product power is clear from (2) via the dependence on the accumulated phase matching along the system given as: $\left|1 /\left[-\alpha+i \Delta \beta_{\text {span }}\right]+1 /\left[g_{1}+i \Delta \beta_{1}\right]+1 /\left[g_{2}+i \Delta \beta_{2}\right]\right|^{2}$. As seen from Fig. 11, using HNLF in the second stage of the DRA results in a slow degradation in the Kerr product power peaks to reach $9 \mathrm{~dB}$ (degradation of peaks) over the $20 \mathrm{GHz}$ frequency separation range, in contrast to the other three cases (6km IDF, $5 \mathrm{~km} \mathrm{DCF}$, and $10 \mathrm{~km} \mathrm{SMF}$ ) that have $18 \mathrm{~dB}$ (degradation of peaks) over the same range. This difference is due to the low dispersion of HNLF compared to the other types of fibres, and that leads to higher nonlinear interference when deploying the HNLF-based second stage of the DRA. 
The performance ( $\mathrm{Q}$ factor, BER, or EVM) of the received optical modulated signals is dependent on the SNR of the modulated signals arriving at the receiver side $[38,39]$.

$$
S N R=\frac{I_{S}}{N h v\left(N F e^{(\alpha L)}-1\right)+\frac{1}{2} I_{S}^{3} \int_{-B_{w} / 2}^{B_{w} / B_{w} / 2} \int_{w}^{B_{w} / 2} \frac{P_{N L}}{P_{S}^{3}} d f_{1} d f_{2}}
$$

$S N R$ is defined as the ratio between the signal power spectral density and the accumulated noise (linear noise and nonlinear Kerr interference) along the system as given in (3). Here $I_{s}$ is the modulated signal power spectral density, $N$ is the number of amplifier spans, $h$ is the Planck's constant, $v$ is the optical frequency, and $N F$ is the noise figure of the dual stage DRA deployed in the system. The first term in the denominator of (3) represents the power spectral density of the linear noise (ASE+DRS) generated from the DRAs, while the second term represents the nonlinear Kerr nonlinear noise generated along the system. The nonlinear noise can be calculated by performing the double integration of the normalised nonlinear Kerr product power (presented in (2)) over the bandwidth of the modulated signals propagating along the system, assuming flat top (Nyquist) WDM signal. Note, the scaling factor of 0.5 represents the correction of double counting of Kerr products along the double integration [40, 41(Eq. 4.1)].

Fig. 12 shows the calculated SNR using (3) for different signal powers per channel within a transmission system consisting of 10 fibre spans of $100 \mathrm{~km}$ SMF and $1 \mathrm{THz}$ bandwidth of Nyquist-WDM signal with test signal centred at $1550 \mathrm{~nm}$. The transmission system deploys dual stage DRAs with the four cases studied in Fig. 9 and Fig. 11. The NF was considered as $6 \mathrm{~dB}$ at $1550 \mathrm{~nm}$ for all the dual stage DRAs schemes (Fig. 8(a)). At low signal powers $(<-6 \mathrm{dBm} /$ channel), similar SNR values were obtained for all the cases because of the same NF performance and low nonlinear penalties. At higher signal powers, SNR performances were degraded by the nonlinear penalties from the different dual stage DRAs due

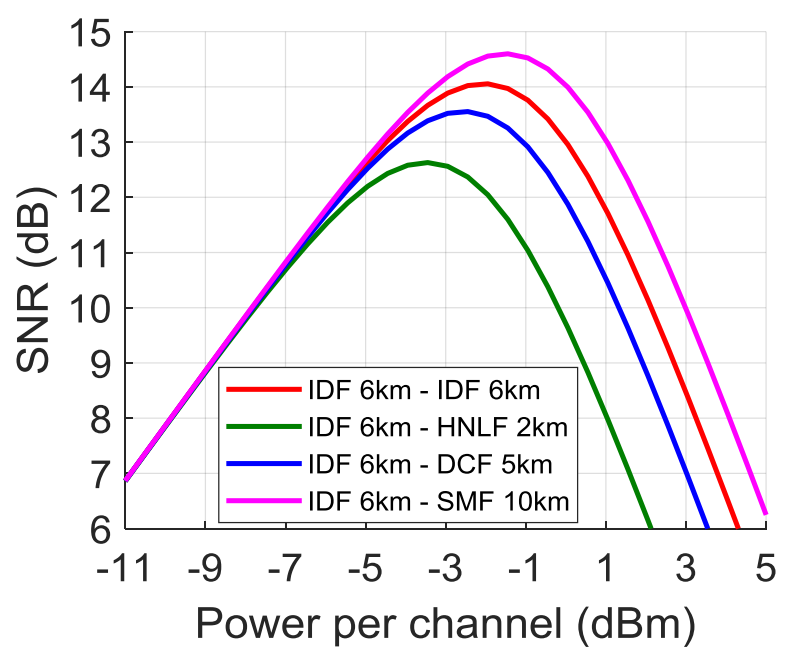

Fig. 12. SNR vs. launch power per channel comparison among different dual stage DRA schemes with different second stage gain fibres to the different gain fibres used in the second stage. Fig. 12 shows that the maximum SNR achieved by the system that deploys HNLF in the second stage DRA is at least $\sim 1 \mathrm{~dB}$ lower than the maximum SNR achieved by the other three systems. The highest SNR (14.6dB) is realised for the system using DRA with SMF in the second stage, which is to be expected as the nonlinear coefficient of the SMF has the lowest value among the fibres in this comparison. At maximum SNR, the optimum launch power per channel was also improved by $0.5 \mathrm{~dB}, 1 \mathrm{~dB}$ and $2 \mathrm{~dB}$ using SMF $(10 \mathrm{~km})$ in the second stage compared with IDF $6 \mathrm{~km}$, DCF $5 \mathrm{~km}$ and HNLF $2 \mathrm{~km}$ respectively. The calculations of SNR were obtained based on the nonlinear Kerr product power calculated for simple piecewise signal power evolution along the DRAs. Increasing the bandwidth would logarithmically decrease the values of the SNR in all the DRA schemes because of additional nonlinear interference from the increased number of signal channels. However, the relative improvement by using IDF $6 \mathrm{~km}$ - SMF 10km dual stage DRA would remain similar with respect to other dual stage DRA configurations as discussed above.

We also calculated the SNR for different transmission distances at the optimum launch power (from Fig. 12) for respective dual stage DRA schemes as shown in Fig. 13. As expected, the dual stage DRA with SMF 10km second stage showed the highest SNR for all distances up to $4000 \mathrm{~km}$. If we consider, Nyquist 32GBaud PM-QPSK modulation format with a HD-FEC limit of $\mathrm{Q}^{2}=\mathrm{SNR}=8.5 \mathrm{~dB}$ then the maximum transmission distance obtained by the IDF $6 \mathrm{~km}-\mathrm{SMF} 10 \mathrm{~km}$ DRA is $3400 \mathrm{~km}$ having extended transmission reach of $8.8 \%$ $(300 \mathrm{~km}), 16.2 \%(550 \mathrm{~km})$ and $27.9 \%(950 \mathrm{~km})$ compared with the IDF $6 \mathrm{~km}-$ IDF $6 \mathrm{~km}$, IDF $6 \mathrm{~km}$ - DCF $5 \mathrm{~km}$ and IDF $6 \mathrm{~km}-$ HNLF $2 \mathrm{~km}$ DRA schemes, respectively. So, the calculated SNR from the NF and proposed analytical model for nonlinear noise power generated in transmission link can predict the transmission system performances based on different dual stage DRA configurations and help to obtain low transmission penalties by choosing the optimum gain fibre properties in

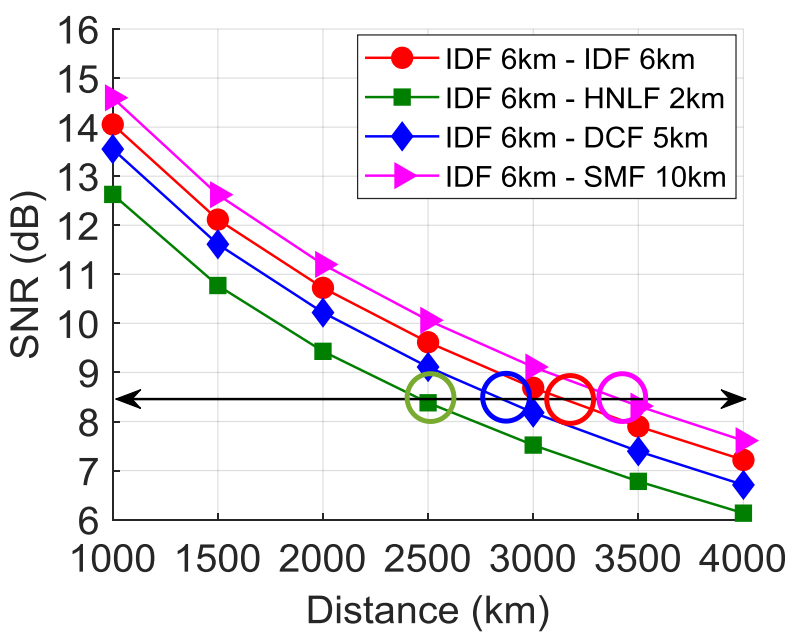

Fig. 13. Comparison of SNR vs. transmission distances at optimum launch power per channel among different dual stage DRA configurations 
each stage. A similar theoretical model for single stage DRA based transmission systems has recently been presented in [42] to predict the performances of broadband optical networks and a reasonable agreement has been shown between the theoretical and experimental results.

\section{CONCLUSIONS}

In this paper, we have characterised the performances of dual stage DRA schemes with different Raman gain fibre combinations with respect to linear noise (ASE and DRS induced MPI) and Kerr induced nonlinearity, through extensive numerical modelling. Of the fibres considered, IDF has been found to be the best gain fibre in the first stage considering the combined penalties from NF and NPS. In $20 \mathrm{~dB}$ net gain dual stage DRAs with fixed IDF $6 \mathrm{~km}$ first stage, a SMF second stage based DRA gives lower nonlinear Kerr product power when compared with conventional Raman gain fibres i.e. IDF, DCF and HNLF.

Finally, in numerical analysis of a $10 \times 100 \mathrm{~km}$ SMF-based transmission system amplified by dual stage DRAs, an IDF $6 \mathrm{~km}$ - SMF $10 \mathrm{~km}$ (first - second stage) based DRA configuration provides the best $\mathrm{SNR}$ of $14.6 \mathrm{~dB}$ giving the following SNR improvements: $0.5 \mathrm{~dB}$ (IDF $6 \mathrm{~km}$ ), $1 \mathrm{~dB}$ (DCF $5 \mathrm{~km}$ ) and $2 \mathrm{~dB}$ (HNLF $2 \mathrm{~km}$ ), due to overall lower nonlinear penalties. Moreover, the IDF 6km - SMF 10km DRA extends the reach of similar 32GBaud Nyquist PM-QPSK based transmission systems up to $3400 \mathrm{~km}$, providing $550 \mathrm{~km}$ and $950 \mathrm{~km}$ reach enhancement with respect to conventional DCF and HNLF based schemes, respectively. We have also shown that the pump power efficiency of the SMF based DRA can be improved by about $50 \%$ by replacing SMF (10km) with IDF $(6 \mathrm{~km})$ in the second stage at a cost of only $0.5 \mathrm{~dB}$ SNR degradation at optimum launch power point.

\section{ACKNOWLEDGMENT}

Original data for this work is available through Aston Research Explorer: (https://doi.org/10.17036/researchdata.aston.ac.uk.00000395).

\section{REFERENCES}

[1] C. Headley and G. P. Agrawal, Raman Amplification in Fiber Optical Communication Systems. Academic Press, 2005.

[2] M. N. Islam, "Raman amplifiers for telecommunications," IEEE J. Sel. Top. Quantum Electron. vol. 8, pp. 548-559, 2002.

[3] Y. Emori, K. Tanaka, and S. Namiki, "100 nm bandwidth flat-gain Raman amplifiers pumped and gain-equalized by 12 -wavelengthchannel WDM laser diode unit", Electron. Lett., vol. 35, pp. 1355 1356, Aug. 1999.

[4] H. Kidorf, K. Rottwitt, M. Nissov, M. Ma, and E. Rabarijaona, "Pump interactions in a 100-nm bandwidth Raman amplifier", IEEE Photon. Technol. Lett., vol. 11, pp. 530-532, May 1999.

[5] L. Grüner-Nielsen and Y. Qian, "Dispersion-compensating fibers for Raman applications," in Raman Amplifiers for Telecommunications 1, ed: Springer, 2004, pp. 161-189.

[6] P. B. Hansen, G. Jacobovitz-Veselka, L. Gruner-Nielsen, and A. Stentz, "Loss compensation in dispersion compensation fiber modules by Raman amplification," in Proc. Opt. Fiber Commun. Conf. Opt. Soc. Amer., 1998, paper TuD1.

[7] Y. Emori, Y. Akasaka, and S. Namiki, "Broadband lossless DCF using Raman amplification pumped by multichannel WDM laser diodes," Electron. Lett., vol. 34, pp. 2145-2146, 1998.
[8] M. Takahashi, R. Sugizaki, J. Hiroishi, M. Tadakuma, Y. Taniguchi, and T. Yagi, "Low-loss and low-dispersion-slope highly nonlinear fibers," $J$. Lightw. Technol., vol. 23, pp. 3615-3624, 2005.

[9] K. Mukasa et al., "Dispersion compensating fiber used as a transmission fiber: inverse/reverse dispersion fiber," J. Opt. Fiber Commun., vol. 3, no. 5, pp. 292-339, Oct. 2006.

[10] I. Nasieva et al., "Nonlinearity management in fiber links with distributed amplification," in Electron. Lett., vol. 39, no. 11, pp. 856-857, May 2003.

[11] P. Parolari, L. Marazzi, L. Bernardini and M. Martinelli, "Double Rayleigh scattering noise in lumped and distributed Raman amplifiers," in J. Lightw. Technol., vol. 21, no. 10, pp. 2224-2228, Oct. 2003.

[12] V. Kalavally, I. D. Rukhlenko, M. Premaratne, "Combined Effect of ASE and DRBS on Noise in Pulse-Pumped Fiber Raman Amplifiers", $J$. Lightw. Technol., vol. 30, no. 18, pp. 2983-2987, 2012.

[13] Y. Emori, Y. Taniguchi, R. Sugizaki, T. Yagi, M. Sakano, and L. E. Nelson, "Impact of Self- and Cross-Phase Modulation on Highly Efficient Discrete Raman Amplifier for 10-Gb/s 100-GHz-Spaced WDM Transmission," in Proc. Optical Amplifiers and Their Applications, Technical Digest (CD) (Optical Society of America, 2005), paper MD2.

[14] H. J. Thiele, J. Bromage and L. Nelson, "Impact of Discrete Raman Amplifier Architecture on Nonlinear Impairments," in Proc. Euro. Conf. Opt. Commun., 2002, paper S.3.09.

[15] D. Hamoir, J. Boniort, L. Gasca, and D. Bayart, "Optimized, two-stage architecture for Raman amplifiers," in Proc. Optical Amplifiers and Their Applications, OSA Trends in Optics and Photonics (Optical Society of America, 2000), paper OMD8.

[16] S. A. E. Lewis, S. V. Chernikov, and J. R. Taylor, "Rayleigh noise suppression using a gain flattening filter in a broadband Raman amplifier," in Proc. Opt. Fiber Commun. Conf., 2000, paper FF5.

[17] S. A. E. Lewis, S. V. Chernikov and J. R. Taylor, "Characterization of double Rayleigh scatter noise in Raman amplifiers," IEEE Photon. Technol. Lett., vol. 12, no. 5, pp. 528-530, May 2000.

[18] A. J. Stentz, S. G. Grubb, C. E. Headley, J. R. Simpson, T. Strasser, and N. Park, "Raman amplifier with improved system performance," in Proc. Opt. Fiber Commun. Conf., 1996, paper TuD3.

[19] M. A. Iqbal, L. Krzczanowicz, P. Skvortcov, A. El-Taher, I. D. Philips, W. Forysiak, J. D. Ania-Castañón and P. Harper, "Performance characterization of high gain, high output power and low noise cascaded broadband discrete Raman amplifiers," in Proc. of 19th International Conference on Transparent Optical Networks (ICTON), 2017, paper WeD5.4.

[20] S. Namiki and Y. Emori, "Ultrabroad-band Raman amplifiers pumped and gain-equalized by wavelength-division-multiplexed high-power laser diodes," IEEE Journal of Selected Topics in Quantum Electronics, vol. 7, no. 1, pp. 3-16, Jan/Feb 2001.

[21] M. A. Iqbal, M. Tan, L. Krzczanowicz, A. E. El-Taher, W. Forysiak, J. D. Ania-Castañón, and P. Harper, "Noise and transmission performance improvement of broadband distributed Raman amplifier using bidirectional Raman pumping with dual order co-pumps," Opt. Express, vol. 25, pp. 27533-27542, 2017.

[22] M. Takahashi, R. Sugizaki, J. Hiroishi, M. Tadakuma, Y. Taniguchi, and T. Yagi, "Low-loss and low-dispersion-slope highly nonlinear fibers," $J$. Lightwave Technol., vol. 23, pp. 3615-3624, Nov. 2005.

[23] L. Grüner-Nielsen and Y. Qian, "Dispersion-compensating fibers for Raman applications," Islam M.N. (eds) Raman Amplifiers for Telecommunications 1, Springer Series in Optical Sciences, vol. 90/1. Springer, New York, NY, 2004.

[24] K. Mukasa, K. Imamura, I. Shimotakahara, T. Yagi, and K. Kokura, "Dispersion compensating fiber used as a transmission fiber: inverse/reverse dispersion fiber," J. Opt. and Fiber Commun. Reports, vol. 3, p. 292, 2006.

[25] J. Bromage, "Raman amplification for fiber communications systems," $J$. of Lightw. Technol., vol. 22, no. 1, pp. 79-93, Jan. 2004.

[26] R. -. Essiambre, P. Winzer, J. Bromage and C. H. Kim, "Design of bidirectionally pumped fiber amplifiers generating double Rayleigh backscattering," IEEE Photon. Technol. Lett., vol. 14, no. 7, pp. 914-916, July 2002.

[27] P. Xiao, Q. Zeng, and J. Huang, "A new optimal algorithm for multi pump sources of distributed fiber Raman amplifier," IEEE Photon. Technol. Lett., vol. 15, pp. 206-208, Feb. 2003.

[28] V. E. Perlin and H. G. Winful, "Optimal design of flat-gain wide-band fiber Raman amplifier," J. Lightwave Technol., vol. 20, pp. 250-254, Feb. 2002. 
[29] S. Cui, J. Liu and X. Ma, "A novel efficient optimal design method for gain-flattened multi wavelength pumped fiber Raman amplifier," IEEE Photon. Technol. Lett., vol. 16, no. 11, pp. 2451-2453, Nov. 2004.

[30] S. Kado, Y. Emori, S. Namiki, N. Tsukiji, J. Yoshida, and T. Kimura, "Broadband flat-noise Raman amplifier using low-noise bidirectionally pumping sources," in Proc. Eur. Conf. on Opt. Commun., 2001, paper PD.F.1.8.

[31] D. M. Baney, P. Gallion, R. S. Tucker, "Theory and measurement techniques for the noise figure of optical amplifiers", Opt. Fiber Technol., vol. 6, no. 2, pp. 122-154, 2000.

[32] G. P. Agrawal, Fiber-Optic Communication Systems, USA, NY, New York: Wiley, pp. 156-158, 2002.

[33] K. O. Hill, D. C. Johnson, B. S. Kawasaki, and R. I. MacDonald, "CW three-wave mixing in single-mode optical fibers," J. Appl. Phys., vol. 49, no. 10 , pp. $5098-5106,1978$.

[34] A. D. Ellis and W. A. Stallard, "Four wave mixing in ultra long transmission systems incorporating linear amplifiers," in Proc. IEE Colloquium on Non-Linear Effects in Fiber Communications, London, 1990, pp. 6/1-6/4.

[35] K. Inoue, "Phase-mismatching characteristic of four-wave mixing in fiber lines with multistage optical amplifiers," Opt. Lett., vol. 17, no. 11, pp. 801-803, 1992.

[36] M. A. Z. Al-Khateeb, M. McCarthy, C. Sánchez, and A. D. Ellis, "Nonlinearity compensation using optical phase conjugation deployed in discretely amplified transmission systems," Opt. Express, vol. 26, pp. 23945-23959, 2018.

[37] M. A. Z. Al-Khateeb, M. Tan, M. A. Iqbal, M. McCarthy, P. Harper and A. D. Ellis, "Four wave mixing in distributed Raman amplified optical transmission systems," in Proc. IEEE Photonics Conference (IPC), Waikoloa, HI, 2016, paper ThB1.1.

[38] R. Schmogrow, B. Nebendahl, M. Winter, A. Josten, D. Hillerkuss, S. Koenig, and J. Meyer, "Error vector magnitude as a performance measure for advanced modulation formats," IEEE Photon. Technol. Lett. vol. 24, pp. 61-63, 2012.

[39] B. Nebendahl, R. Schmogrow, T. Dennis, A. Josten, D. Hillerkuss, S. Koenig, J. Meyer, M. Dreschmann, M. Winter, M. Huebner, W. Freude, C. Koos, J. Leuthold, "Quality Metrics in optical modulation analysis: EVM and its relation to Q-factor, OSNR, and BER," in Proc. Asia Communications and Photonics Conference (ACP), Guangzhou, 2012, paper AF3G.2.

[40] X. Chen and W. Shieh, "Closed-form expressions for nonlinear transmission performance of densely spaced coherent optical OFDM systems," Opt. Express, vol. 18, no. 18, pp. 19039-54, 2010.

[41] M. Al-Khateeb, "Experimental demonstration of performance enhancement in non-linearity limited optical fibre systems," Thesis (Ph.D.)--Aston University, 2018. http://publications.aston.ac.uk/37785/

[42] L. Krzczanowicz, M. Al-Khateeb, M. A. Iqbal, I. D. Phillips, P. Harper, W. Forysiak, "Performance estimation of discrete Raman amplification within broadband optical networks" in Proc. Opt. Fiber Commun. Conf., 2019, paper Tu3F.4.

Md Asif Iqbal received the B.Sc. degree in electrical, electronic and communication engineering from the Military Institute of Science and Technology (MIST), Dhaka, Bangladesh, in 2010, the M.Sc. degree in photonic networks engineering from Scuola Superiore SantAnna, Pisa, Italy, and Aston University, Birmingham, U.K., in 2014, and the Ph.D. degree in electronic engineering from the Aston University, Birmingham, U.K., in 2018. $\mathrm{He}$ is currently a postdoctoral research fellow with the Aston Institute of Photonic Technologies (AIPT) at Aston University where he is working on advanced Raman amplification techniques for long-haul and ultra-wideband coherent optical communication systems.

Mohammd Al-Khateeb is a Senior Hardware Development Engineer at Infinera Corporation. He received double MSc degree in photonics networks engineering (MAPNET) from Scuola Superiore Sant'Anna and Aston University, in 2014. Mohammad has received his Ph.D. degree in 2018 from Aston University under the supervision of Prof. Andrew Ellis, researching the ability to expand the capacity of optical fibre transmission systems through nonlinearity compensation techniques. He has authored/co-authored over 37 journal and conference papers on nonlinearity compensation techniques (digital signal processing or all-optical compensation) to minimize impact of nonlinearities on the performance of optical fibre transmission systems.

Lukasz Krzczanowicz received his M.Sc. degree in "Optoelectronics and Optical Waveguide Technology" from Wrocław University of Science and
Technology (Department of Microsystem Electronics and Photonics), Poland in 2008 and Ph.D. in Optical Communications from University of Limerick (Department of Electronic and Computer Engineering), Ireland in 2015, where he was working on Optical Phase Modulated Data Amplification and Wavelength Conversion using Quantum-Dot Semiconductor Optical Amplifiers (QD-SOAs). He is currently a postdoctoral research fellow with the Aston Institute of Photonic Technologies (AIPT) at Aston University, Birmingham, UK where he is working on Raman amplification techniques for next generation, ultra-wideband optical communications systems.

Ian D Phillips received his Ph.D. degree in optical communications from Aston University, Birmingham, UK in 1997 for his study of high-speed alloptical regeneration. He continued his research career in optical communications at British Telecom Research Laboratories and later Corning Research Centre. In 2000 he joined Marconi-Solstis working on ultra-long haul (3000km) DWDM transmission where his worked focused on transceiver design. He continued this work at Marconi, and later at Ericsson before moving into a broader role of Photonic System Test based in Genoa, Italy. He returned to Aston University in 2012 as a Research Fellow where his worked focused on compensating nonlinear transmission effects with an overall goal to increase system capacity.

Paul Harper receiver the Ph.D. degree in ultra-long haul dispersion managed soliton transmission from Aston University in 1997. He continued as Aston University as a Post-Doctoral Research Fellow until moving to industry as a founding member of the start-up Marconi-Solstis in 2000 developing optical communications systems based on dispersion managed soliton techniques. In 2005 he returned to Aston University where he has led extensive research on advanced Raman amplification techniques for high capacity optical communications.

Wladek Forysiak holds a Ph.D. in physics and has a research background in nonlinear photonics and high-speed optical fibre communication systems. He was a co-founder of Marconi Solstis in 2000 and spent 15 years in WDM system related product development with Marconi, Ericsson, and Oclaro. His current research interests are in wideband optical fibre communication systems, optical devices and subsystems, and the impact and mitigation of device and fibre nonlinearities. 